\title{
SI-2a
}

\section{Review of Condition Based Maintenance approaches for vapor compression refrigeration systems}

\author{
from Ron van de Sand ${ }^{1}$, Sandra Corasaniti², Jörg Reiff-Stephan ${ }^{3}$
}

\section{Abstract}

Vapor compression refrigeration systems are subject to performance degradation over time due to the presence of faults. However, latest work in the field of condition-based maintenance shows promising results in the automatic early detection of anomalous behaviour as well as in accurate machine diagnostics and can, therefore, increase the overall system reliability by simultaneously preventing machine downtimes. In this paper, the latest research works carried out within the last decade are reviewed and the approaches are classified regarding their working principles. Furthermore, the work at hand depicts the current research trend in this field and outlines current obstacles.

\section{Introduction}

Vapor compression refrigeration systems (VCRS) are used in many industrial and commercial applications and are considered as large energy consumers. Particularly in the food industry, but also in other branches, such as the chemical or pharmaceutical industries, these systems are subject to high reliability standards and can account for $20 \%-40 \%$ of a facility's energy consumption [2] depending on the application scenario. Consequently, this sector offers particularly high potential for optimisation in terms of energy savings and reliability considerations. As the overall system performance is often decreased due to the presence of faults, energy efficient systems should be equipped with a condition monitoring system in order to enable the online system assessment of the respective appliance. Based on this measure, system faults may automatically be detected and diagnosed, thus, preventing energy waste and high maintenance costs [3]. Therefore, Condition-Based Maintenance (CBM) techniques have attracted attention for decades, and many approaches are well described across the literature. Due to CBM, maintenance actions can be scheduled based on the actual system condition [4] rather than on predetermined time intervals leading to lower operating costs [5]. Moreover, as VCRS can lose about $30 \%$ efficiency due to degradation processes by simultaneously seeming fully functional [6], CBM enables to detect and diagnose faults at an early stage of development and can, therefore, avoid energy waste. The main objective of a CBM systems is the automated detection and classification of faults with the lowest possible degree of severity, so that maintenance measures can be initiated in time. Besides the many subsystems of a CBM model, such as data-filtering or feature extraction, most researchers focussed on the development of automatic VCRS degradation assessment strategies. Especially fault detection and diagnosis (FDD) has been treated recently, which is indeed the most critical part in the CBM model development. Therefore, the work at hand reviews research contributions to the field with a special view to fault detection and

\footnotetext{
${ }^{1}$ Ron van de Sand, M. Eng., Technische Hochschule Wildau

2 Sandra Corasaniti, Ph. D., University of Rome "Tor Vergata"

${ }^{3}$ Prof. Dr.-Ing. Jörg Reiff-Stephan, Technische Hochschule Wildau
} 
diagnosis for VCRS published within the last decade and points out promising results. Furthermore, this paper provides an overview of the most common mythologies applied and describes the current state of the art.

After this introduction, the paper is organized as follows; Section 3 outlines the field of CBM and describes the implementation of FDD as a prerequisite for machine prognostics and summarizes the model approaches in regards to their operating principle. In section 4, the research work of the last decade in this field is reviewed and subsequently summarized in section 5 .

\section{Conditon Based Maintanance}

\subsection{Terms and Definitions}

The term Conditon Based Maintanance is defined within the ISO 13372 [7] and replaces the older term Predictive Maintenance (PM), which is still commonly used across the literature. In many cases, authors use both terms equivalently and no difference is made between machine diagnostics and prognostics. However, as accurate machine diagnostic is a prerequisite for the implementation of a prognostic model, that may be applied for estimating the Remaining-UsefulLife (RUL) of a machine or component, the work at hand follows the classification suggested in [1]. As illustrated in Figure 1, PM can be divided into CBM and Prognostic Health Management (PHM), whereas PHM primarily concerns life cycle management and the prediction of future events [8], such as the fault evolution. CBM, on the other hand, aims to assess the current condition of a machine by use of run-time data and to determine its current fault/failure state [1]. It is generally understood that such a model can correctly diagnose occurring faults as well as estimating their severity level [9].

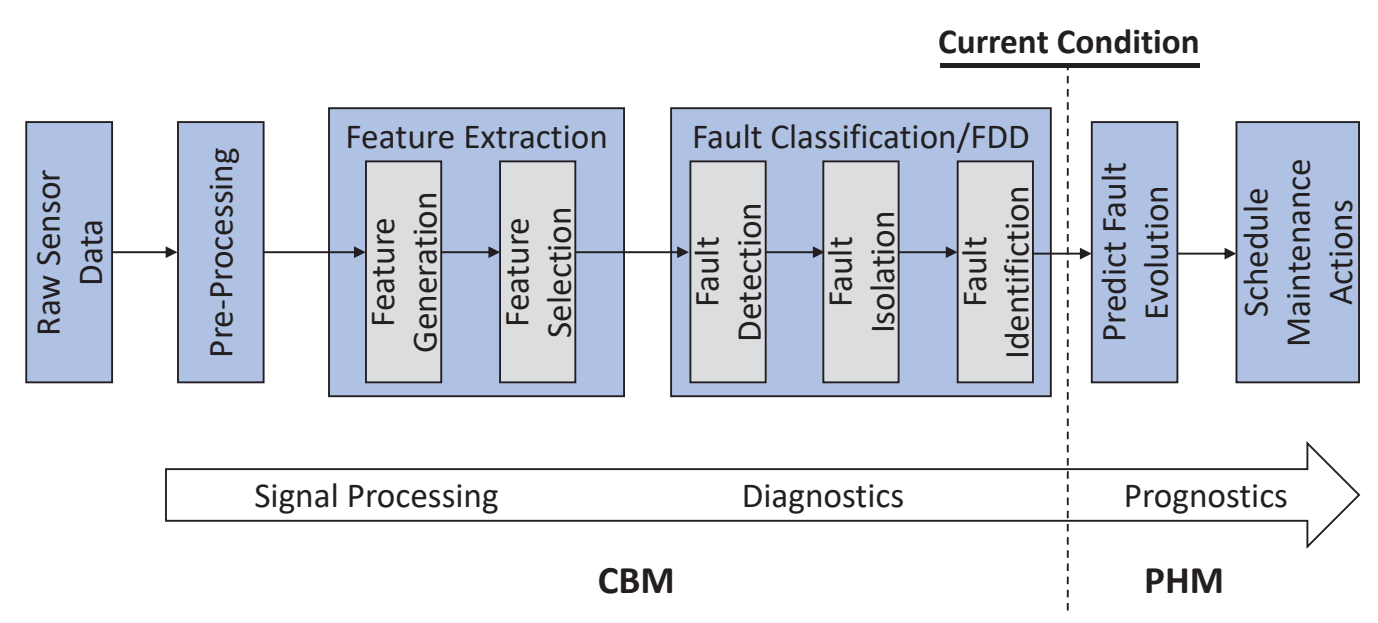

Figure 1 CBM - PHM cycle [1]

A CBM model constitutes three consecutive steps, namely; data pre-processing, feature extraction and fault classification. The former step is primarily applied for increasing the signal-to-noise ratio [8] of the collected data in order to improve the prediction accuracy of the respective fault model. It is worth noticing that this step could also include the detection of the stationary system state, which is especially important for VCRS. Such steady-state detectors are often employed in order to exclude data stemming from transient system states (for example during start-up or 
shutdown phases) as such may not represent the actual system state and consequently lead to inadequate machinery health assessment. The objective of the second step is to extract characteristic features (CF) [9], which indicate the presence of a fault and enable to distinguish multiple fault patterns from one another. During the feature generation phase, such features can either directly be obtained from sensor readings or can be computed through arithmetic operations as well as thermodynamic dependencies [9], such as the coefficient of performance or the condenser/evaporator heat flow. In the following step, fault indicative CFs are selected to improve the overall FDD accuracy of the subsequent model by rejecting features with low fault sensitivity as well as redundant features. Generally, feature selection is a crucial step within the CBM model development as it significantly impacts its diagnostic capabilities. Thus, several methods have been proposed in recent years, for instance the Relief+AGA approach proposed by Yan et al. [3]. The final phase includes the actual fault classification, which we refer to as FDD in the following. In the first instance, fault detection is applied though anomaly recognition methods in order to identify abnormal behaviour. Subsequently, fault isolation is applied in order to identify the failing component or subsystem and is followed by fault identification which is used to determine the fault origin [1] as well as its severity. Although all steps are indeed important in order to develop a highly reliable CBM model, most researchers focussed specifically on the development of FDD strategies, as this constitutes the most challenging part. Therefore, the work at hand reviews research contributions to this field of research published within the last decade and points out promising results.

\subsection{Approaches}

FDD methods can generally be classified into two categories: model-based and data-driven [913]. The major difference between both methods is that the former relies on a dynamical reference model of the observed system, whereas data-driven ones make use of historical data [1] for machinery health assessment. It goes without saying that both categories come with their respective pros and cons. Most of all, model-based FDD systems may even detect unanticipated faults as they consider the residuals between a model $F(\cdot)$ and the actual system parameters. The presence of a fault is consequently indicated by rising residuals between both outputs. On the other hand, the definition of a mathematical formulation of a physical system may be difficult or even infeasible for systems with high complexity, which seems to be a drawback of such white-box models. This still constitutes a major challenge for VCRS as modelling multi-phase flows inside the refrigerant cycle, especially for complex geometries, can be challenging. Blackbox models [10,14-17] in turn, do not depend on the underlying physical structure of a system, but rather are based on learning the relationships between input and output variables of $F(\cdot)$ using statistical and machine learning methods. As shown in Figure 2, another widely applied approach is to apply data-driven models enabling learning of fault patterns on the basis of historical machine data.

According to Dai and Gao [13], such models can be further subdivided into qualitative and quantitative methods, whereas the former applies symbolic intelligence, which can be expressed through domain knowledge in form of, for example, fault pattern libraries, fuzzy expert systems [1] or fault trees [13]. In contrast, quantitative models aim to learn underlying fault patterns from historical data in order to enable FDD in an automatic fashion. This is further classified into supervised (learning with target labels) [9, 11, 12, 18-28], unsupervised (learning without target labels) and semi-supervised (learning from partly labelled data) [3]. Reinforced-learning models have also recently attracted attention as they enable to learn from trial-and-error by repetitively 
receiving awards. However, in the considered field of application, no successful reinforcedlearning approaches are known, so that their practicability for FDD purposes remains questionable. A major drawback of data-driven approaches may lie in the low availability of datasets containing fault samples, as faults are commonly rare events [29]. Furthermore, the fault isolation capabilities of these models are often limited to anticipated faults [1] and therefore adding new faults to the FDD model requires associated data to retrain it.

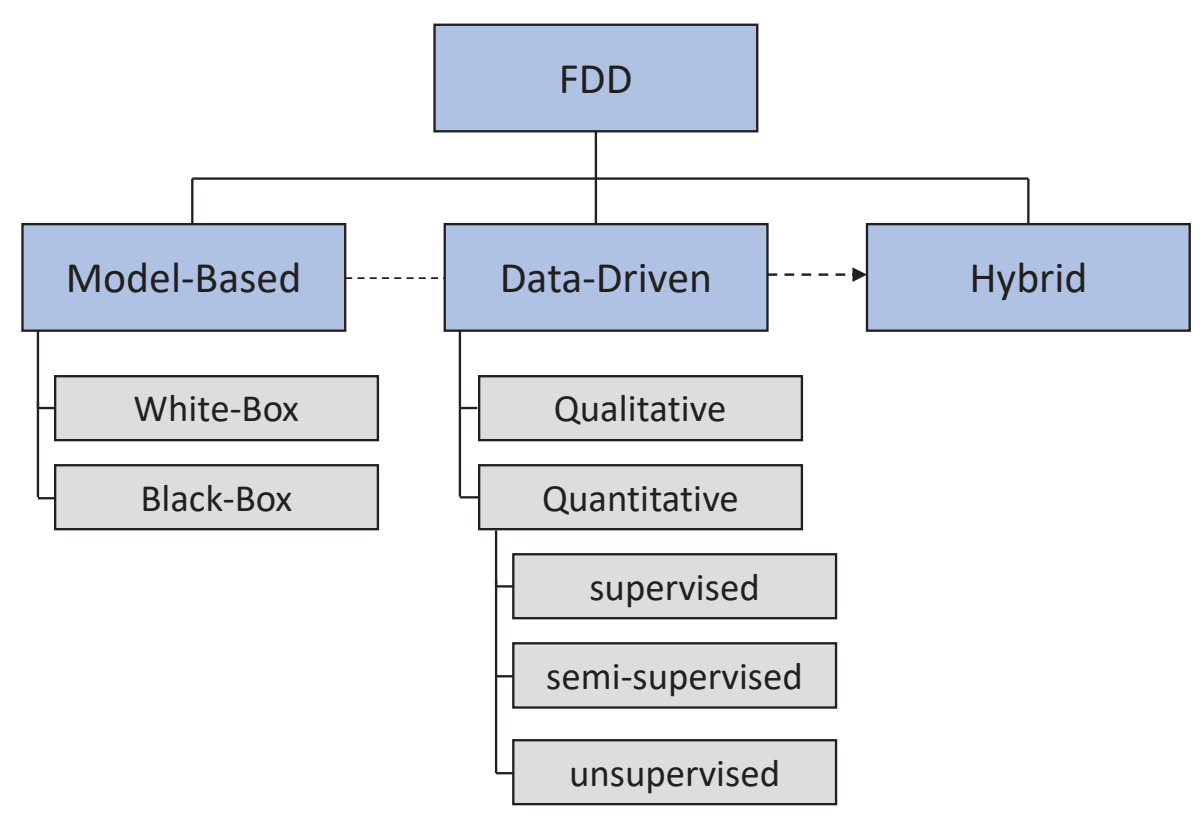

Figure 2 Overview FDD Approaches

Besides, authors have also tried to implement hybrid models $[3,30]$ by combining both modelbased and data-driven methods. Following this classification, it can be concluded that the designation of whether an FDD approach is model-based, data-driven, or hybrid is reasonable for the fault detection phase as faults isolation is always conducted in a data-driven fashion. For example, in most of the reviewed model-based systems, faults are detected if the residuals between the reference model and the monitored process exceed predefined limits and the fault is then classified using decision tables. As a consequence, this can clearly be determined as being qualitative data-driven. Other fault isolation methods may also apply quantitative fault classification algorithms, such as support vector machines (SVM) or artificial neural networks. Therefore, the rest of the paper refers only to the classification of the working principle regarding the fault detection phase.

\section{Review}

\subsection{Model-Based}

Although most works carried out during the last decade utilized black-box models for their modelbased FDD approach, some researchers developed white-box models as well. Zhao et al. [31], proposed a simplified physical-model based on the basic principle of a VCRS for the computation of performance indicators, of which the normalized heat transfer coefficient of the condenser 
is used for fault detection. The residual threshold triggering the alarm is subsequently determined empirically based on the false alarm rate in the fault-free case.

Nonetheless, most of the latest research is based on black-box models, which do not require detailed modelling of the target domain. In [15], the model is developed by applying the reference model based on multiple linear regression (MLR) as proposed by Comstock et al. [32] and an adaptive estimator is used for setting the residual alarm threshold. In [17], the reference model has been replaced by support vector regression and exponentially-weighted moving average (EWMA) control charts are applied to further reduce the false-negative rate. This idea has later been adopted by Tran et al. [14], who showed that a radial basis function (RBF) neural network as a reference model can yield higher fault detection accuracy. Furthermore, the authors compared three different reference models [33] combined with EWMA control charts and showed that the RBF neural network based one achieved the highest accuracy. In the same year, the authors proposed a new reference model [34] based on the least square support vector regression. In conjunction with an evolutionary optimization algorithm for hyperparameter optimisation, the model outperformed the former black-box models regarding their fault detection accuracy. Zhao et al. [16] investigated three reference models for fault detection, namely MLR, simple linear regression, and the decoupling based FDD approach [35], of which the later was shown to be the most effective one. Their research particularly investigated the FDD strategies in the presence of multiple simultaneous faults. Another contribution to the topic is given by Beghi et al. [10], where different multiple-input-single-output models (ARMAX, ARX, etc.) were empirically identified for each selected CF respectively.

Even though the fault detection part varies greatly among the model-based methods, the fault diagnosis approach always appears similar as all contributions utilize decision tables for fault classification purposes. However, some papers applied different fault diagnosis strategies as shown in [27], where faults are detected by the use of residuals generated from the MLR model and diagnosed using Bayesian Belief Network.

\subsection{Data-Driven}

Generally, qualitative data-driven FDD methods are well suitable for diagnosing faults but are mostly limited in terms of detecting abnormal behaviour. Thus, most data-driven fault detection approaches mostly utilize quantitative methods relying on historical data only. In [12], a SVM classifier was utilized to detect and isolate multiple simultaneous faults. Han et al. [21] proposed a SVM based diagnostic tools that combines FDD in one algorithm. They also applied a genetic algorithm to identify CFs and demonstrated how this improved the diagnostic performance. The feature reduction problem has been particularly addressed in great detail across the literature and many researchers have shown significant improvement in the FDD performance when used in conjunction with data-driven classifiers. One widely applied dimensionality reduction method is known to be principal component analysis (PCA), which aims to identify a lower dimensional space of uncorrelated features by decomposing any observation into the modelled principal component subspace and an un-modelled residual subspace [28]. Similar to the proposed SVM diagnostic tool described in [21], Han et al. [19] earlier proposed to apply PCA on the given dataset and to train the SVM on the most significant principal components. A similar idea was also expressed by Zhao et al. [28] who applied a support vector data description (SVDD) clas- 
sifier on the principal components for anomaly detection using only normal (fault-free) data samples in the training phase. In the following year, Beghi et al. [29] applied the same approach, but used the $\vartheta$-OCSVM one class classification algorithm proposed by Schölkopf et al. [36] for fault detection and applied a heuristic approach for choosing the gaussian width parameter of the RBF kernel. It is worth noticing that the SVDD and the $\vartheta$-OCSVM classifier reach equal results when implemented using the RBF kernel [36]. PCA has also been adopted by Li et al. [26], who modelled a SVDD classifier using the residual subspace instead of the principal component subspace. It was furthermore demonstrated that their proposed PCA-R-SVDD algorithm outperformed its principal component counterpart. Another fault detection model is described in [37], where a SVDD classifier is trained for the normal operating class as well as for each investigated fault respectively. Han et al. [20] described the use of a Least Squares Support Vector Machine (LS-SVM) for chiller FDD and compared the approach with a standard SVM and a RBF neural network. Their work demonstrated that by selecting the most significant features, the correct rate of the LS-SVM is slightly higher. Besides the application of the SVM and its derivatives for machine diagnostic purposes, some contributions also described the use of other algorithms. For instance, Wang et al. [23] used a bayesian network in different variations and Li et al. [25] proposed to apply linear discriminant analysis for both fault detection and isolation.

Recently, researchers have particularly addressed the lack of available datasets, as this is still a major problem in the development of quantitative FDD methods. One example is given by Beghi et al. [9], who first applied PCA in conjunction with a statistical model. In their work, faults are further diagnosed through a decision table. In [24], a generative adversarial neural network has been proposed to generate augmented data samples in the presence of only a few real fault samples. The authors demonstrated the effectiveness of their approach by applying a SVM for the automatic classification of faults. Another problem is also the transferability of FDD models across different domains, as data stemming from different refrigeration systems are subject to different distributions. This issue was i.a. treated by Fan et al. [11], who proposed a transferable model from a known source domain (labels are known) to a partly known target domain (only a few fault labels are known). The model transfer could be realised by oversampling the known samples from the target domain using the combined principal component subspace. The authors then applied a SVM to classify the unknown labels of the target domain. Their work was later enhanced in [18].

\subsection{Hybrid}

Although the great variety of model-based and data-driven methods have been shown to reach decent results, few researchers have also tried to combine both. In [3], Yan et al. proposed to apply an extended kalman filter in combination with an ARX model from which 18 parameters could be extracted and fitted recursively into a $\vartheta$-OCSVM classifier in a semi-supervised manner. The authors also suggested a twostep feature selection approach by first reducing the feature space using the ReliefF algorithm and then to reject redundant features by applying the adaptive genetic algorithm. Similarly, in [30] they proposed to use the ARX parameters for the application of a multi class SVM and demonstrated its effectiveness. 


\section{Summery}

Based on the number of reviewed contributions, a clear trend in the development of CBM models for VCRS can be derived. Many authors addressed the development of model-based fault detection strategies and demonstrated promising results. As illustrated in Figure 3, 30\% of the 27 reviewed contributions followed this idea, of which $88 \%$ rely on black-box models using variations of regression or moving average reference models. However, the majority of contributions describe data-driven approaches for FDD. In fact, around $63 \%$ of all contributions in the last ten years are based on such quantitative models, which demonstrates the importance of the topic in this research field. In contrast, only $7 \%$ proposed hybrid systems combining both philosophies.

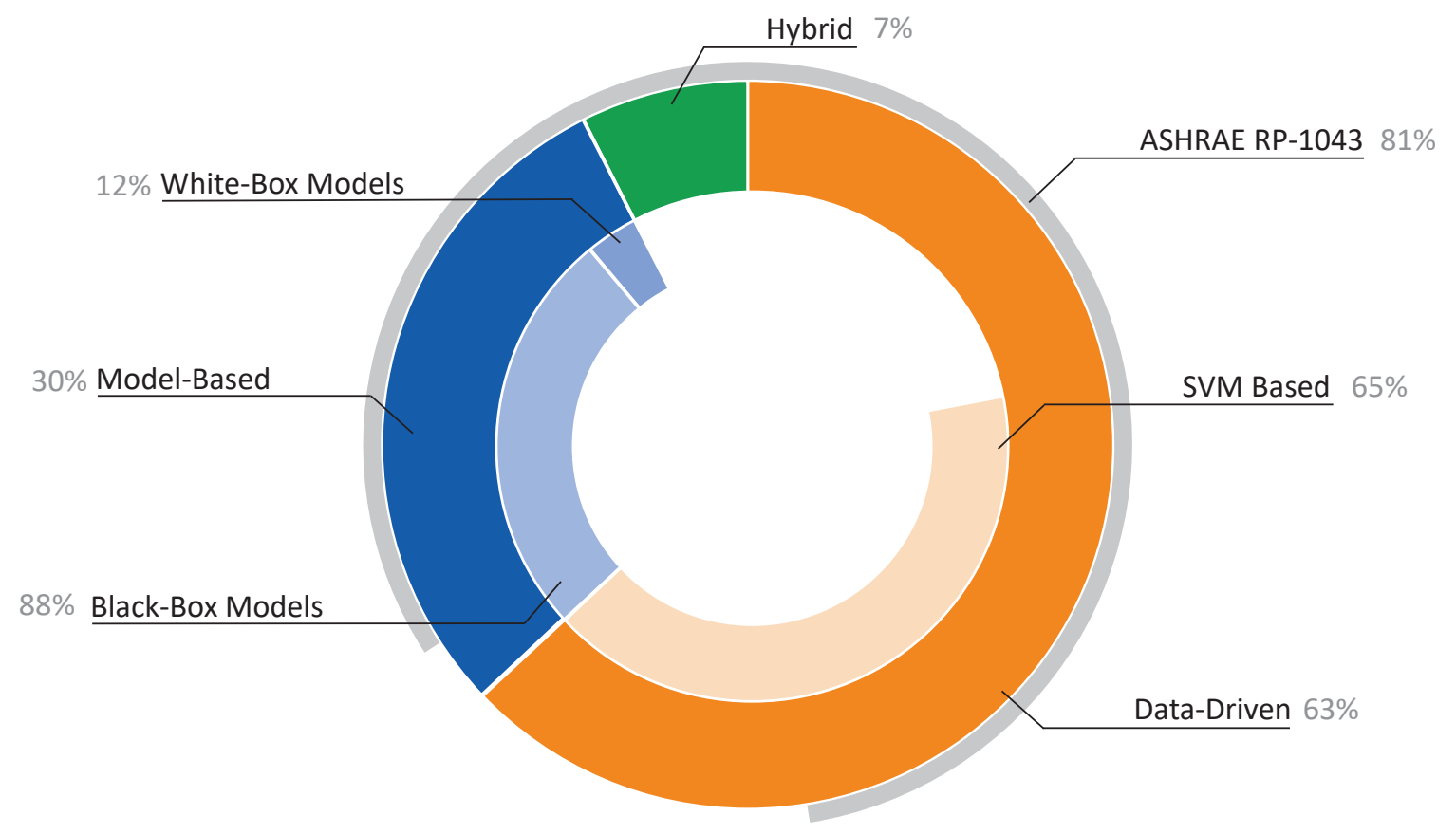

Figure 3 Summery - FDD approaches for VCRS

It is worth noticing, that $65 \%$ of the data-driven models consider the application of the SVM or its derivatives (multi-class SVM, SVDD, $\vartheta$-OCSVM, etc.). Moreover, this algorithm has also been applied in the development of hybrid models as well as for model-based FDD. The later primarily used support vector regression to build a reference model as it can capture non-linear relationships by applying the kernel-trick [38], such as RBF or polynomial kernels. In general, it can be stated that $56 \%$ of all reviewed publications in this field use a modification of the SVM for the respective purposes. The reason for this might be that this algorithm shows goods generalization performance and is quite effective even with a small amount of data available. Furthermore, training is usually conducted by solving a quadratic optimisation problem and is therefore considered to be computationally effective.

In addition, the review at hand shows that a large proportion of the contributions are based on the same dataset. About $81 \%$ of the papers validate their approach solely on the basis of the ASHRAE research project RP-1043 conducted by Comstock et al. [32], where common VCRS faults were investigated using a $360 \mathrm{~kW}$ centrifugal chiller. As mentioned earlier, the lack of available datasets containing a wide range of different fault samples still constitutes a major problem, 
especially for data-driven models. As a result, this may degrade the FDD prediction accuracy as data is commonly subject to different distributions across various domains and, thus, inhibit their broad application. So far, only two contributions concern the transferability of data-driven methods across domains, which enable to transfer knowledge from a source domain using small amount of fault instances stemming from the target domain. However, it must be concluded that no approach is described which enables the transfer of knowledge across domains with unknown target domain labels. Due to the great variety of VCRS, this must be further addressed in future research projects in order to allow the broad application of CBM models and to benefit from their advantages.

\section{Conclusion}

The work at hand reviewed papers published within the last decade concerning CBM approaches for VCRS. Besides the clarification of the classification of all partial aspects of the topic and the classification of these, the current research trend in this field was pointed out. It was shown the majority of the proposed utilize data-driven methods and primarily apply the support vector machine classifier in its numerous variations for both fault detection and fault diagnosis. Moreover, it can be stated that almost all contributions use machine learning methods in different ways. This shows the importance of this topic for this field of research and can, therefore, be seen as an important key enabler. Yet, the low availability of appropriate datasets remains a challenge in the development of CBM models and should therefore also be addressed in the future.

\section{References}

[1] G. J. Vachtsevanos, Intelligent fault diagnosis and prognosis for engineering systems: Wiley Hoboken, 2006.

[2] X. Zhao, "Lab test of three fault detection and diagnostic methods' capability of diagnosing multiple simultaneous faults in chillers," Energy and Buildings, vol. 94, pp. 43-51, 2015.

[3] K. Yan, Z. Ji, and W. Shen, "Online fault detection methods for chillers combining extended kalman filter and recursive one-class SVM," Neurocomputing, vol. 228, pp. 205212, 2017.

[4] Begriffe der Instandhaltung, 13306.

[5] L. Wen, X. Li, L. Gao, and Y. Zhang, "A new convolutional neural network-based datadriven fault diagnosis method," IEEE Transactions on Industrial Electronics, vol. 65, no. 7, pp. 5990-5998, 2017.

[6] S. Wang, Q. Zhou, and F. Xiao, "A system-level fault detection and diagnosis strategy for HVAC systems involving sensor faults," Energy and Buildings, vol. 42, no. 4, pp. 477490, 2010.

[7] ISO, Condition monitoring and diagnostics of machines-Vocabulary, ISO 13372.

[8] C. Anger, "Hidden semi-Markov models for predictive maintenance of rotating elements," Technische Universität, 2018. 
[9] A. Beghi, R. Brignoli, L. Cecchinato, G. Menegazzo, M. Rampazzo, and F. Simmini, "Data-driven fault detection and diagnosis for HVAC water chillers," Control Engineering Practice, vol. 53, pp. 79-91, 2016.

[10] A. Beghi, L. Cecchinato, F. Peterle, M. Rampazzo, and F. Simmini, "Model-based fault detection and diagnosis for centrifugal chillers," in 2016 3rd Conference on Control and Fault-Tolerant Systems (SysTol), 2016, pp. 158-163.

[11] Y. Fan, X. Cui, H. Han, and H. Lu, "Chiller fault diagnosis with field sensors using the technology of imbalanced data," Applied Thermal Engineering, vol. 159, p. 113933, 2019.

[12] H. Han, B. Gu, Y. Hong, and J. Kang, "Automated FDD of multiple-simultaneous faults (MSF) and the application to building chillers," Energy and Buildings, vol. 43, no. 9, pp. 2524-2532, 2011.

[13] X. Dai and Z. Gao, "From model, signal to knowledge: A data-driven perspective of fault detection and diagnosis," IEEE Transactions on Industrial Informatics, vol. 9, no. 4, pp. 2226-2238, 2013.

[14] D. A. T. Tran, Y. Chen, M. Q. Chau, and B. Ning, "A robust online fault detection and diagnosis strategy of centrifugal chiller systems for building energy efficiency," Energy and Buildings, vol. 108, pp. 441-453, 2015.

[15] F. Xiao, C. Zheng, and S. Wang, "A fault detection and diagnosis strategy with enhanced sensitivity for centrifugal chillers," Applied Thermal Engineering, vol. 31, 17-18, pp. 39633970, 2011.

[16] X. Zhao, "Lab test of three fault detection and diagnostic methods' capability of diagnosing multiple simultaneous faults in chillers," Energy and Buildings, vol. 94, pp. 43-51, 2015.

[17] Y. Zhao, S. Wang, and F. Xiao, "A statistical fault detection and diagnosis method for centrifugal chillers based on exponentially-weighted moving average control charts and support vector regression," Applied Thermal Engineering, vol. 51, 1-2, pp. 560-572, 2013.

[18] Y. Fan, X. Cui, H. Han, and H. Lu, "Chiller fault detection and diagnosis by knowledge transfer based on adaptive imbalanced processing," Science and Technology for the Built Environment, pp. 1-18, 2020.

[19] H. Han, Z. Cao, B. Gu, and N. Ren, "PCA-SVM-based automated fault detection and diagnosis (AFDD) for vapor-compression refrigeration systems," HVAC\&R Research, vol. 16, no. 3, pp. 295-313, 2010.

[20] H. Han, X. Cui, Y. Fan, and H. Qing, "Least squares support vector machine (LS-SVM)based chiller fault diagnosis using fault indicative features," Applied Thermal Engineering, vol. 154, pp. 540-547, 2019.

[21] H. Han, B. Gu, J. Kang, and Z. R. Li, "Study on a hybrid SVM model for chiller FDD applications," Applied Thermal Engineering, vol. 31, no. 4, pp. 582-592, 2011.

[22] S. He, Z. Wang, Z. Wang, X. Gu, and Z. Yan, "Fault detection and diagnosis of chiller using Bayesian network classifier with probabilistic boundary," Applied Thermal Engineering, vol. 107, pp. 37-47, 2016.

[23] Z. Wang, Z. Wang, S. He, X. Gu, and Z. F. Yan, "Fault detection and diagnosis of chillers using Bayesian network merged distance rejection and multi-source non-sensor information," Applied Energy, vol. 188, pp. 200-214, 2017.

[24] K. Yan, A. Chong, and Y. Mo, "Generative adversarial network for fault detection diagnosis of chillers," Building and Environment, vol. 172, p. 106698, 2020. 
[25] D. Li, G. Hu, and C. J. Spanos, "A data-driven strategy for detection and diagnosis of building chiller faults using linear discriminant analysis," Energy and Buildings, vol. 128, pp. 519-529, 2016.

[26] G. Li et al., "An improved fault detection method for incipient centrifugal chiller faults using the PCA-R-SVDD algorithm," Energy and Buildings, vol. 116, pp. 104-113, 2016.

[27] Y. Zhao, F. Xiao, and S. Wang, "An intelligent chiller fault detection and diagnosis methodology using Bayesian belief network," Energy and Buildings, vol. 57, pp. 278-288, 2013.

[28] Y. Zhao, S. Wang, and F. Xiao, "Pattern recognition-based chillers fault detection method using support vector data description (SVDD)," Applied Energy, vol. 112, pp. 1041-1048, 2013.

[29] A. Beghi, L. Cecchinato, C. Corazzol, M. Rampazzo, F. Simmini, and G. A. Susto, "A one-class svm based tool for machine learning novelty detection in hvac chiller systems," IFAC Proceedings Volumes, vol. 47, no. 3, pp. 1953-1958, 2014.

[30] K. Yan, W. Shen, T. Mulumba, and A. Afshari, "ARX model based fault detection and diagnosis for chillers using support vector machines," Energy and Buildings, vol. 81, pp. 287-295, 2014.

[31] Y. Zhao, S. Wang, F. Xiao, and Z. Ma, "A simplified physical model-based fault detection and diagnosis strategy and its customized tool for centrifugal chillers," HVAC\&R Research, vol. 19, no. 3, pp. 283-294, 2013.

[32] M. C. Comstock and J. E. Braun, "Development of analysis tools for the evaluation of fault detection and diagnostics in chillers, ASHRAE Research Project RP-1043," American Society of Heating, Refrigerating and Air-Conditioning Engineers, Inc., Atlanta. Also, Report HL, 99-20, 1999.

[33] D. A. T. Tran, Y. Chen, and C. Jiang, "Comparative investigations on reference models for fault detection and diagnosis in centrifugal chiller systems," Energy and Buildings, vol. 133, pp. 246-256, 2016.

[34] D. A. T. Tran, Y. Chen, H. L. Ao, and H. N. T. Cam, "An enhanced chiller FDD strategy based on the combination of the LSSVR-DE model and EWMA control charts," International Journal of Refrigeration, vol. 72, pp. 81-96, 2016.

[35] H. Li and J. E. Braun, "A methodology for diagnosing multiple simultaneous faults in vapor-compression air conditioners," HVAC\&R Research, vol. 13, no. 2, pp. 369-395, 2007.

[36] B. Schölkopf, R. C. Williamson, A. J. Smola, J. Shawe-Taylor, and J. C. Platt, "Support vector method for novelty detection," in Advances in neural information processing systems, 2000, pp. 582-588.

[37] Y. Zhao, F. Xiao, J. Wen, Y. Lu, and S. Wang, "A robust pattern recognition-based fault detection and diagnosis (FDD) method for chillers," HVAC\&R Research, vol. 20, no. 7, pp. 798-809, 2014.

[38] B. Schölkopf, A. J. Smola, and F. Bach, Learning with kernels: support vector machines, regularization, optimization, and beyond: the MIT Press, 2018. 